\title{
TG07
}

\section{Resolving Created, Propped, and Effective Hydraulic Fracture Length}

\section{C.L. Cipolla* (Carbo)}

\section{SUMMARY}

The conclusions from the work are listed below.

1. Fracture modeling (net pressure analysis) can result in non-unique interpretations of fracture geometry if the physics of fracture propagation are uncertain.

$2 . \quad$ Production analyses and reservoir simulation history matching can result in non-unique interpretations of effective fracture length and conductivity in the absence of accurate measurements of reservoir permeability and pressure.

3 . Pre- and post-fracture pressure buildup tests can provide important information to evaluate hydraulic fracture performance.

4. Microseismic and/or tilt fracture mapping can provide direct measurements of created fracture length and fracture complexity, but cannot provide insights into the propped and effective fracture length. 5. Multiple fracture diagnostic technologies are required to reliably evaluate hydraulic fracture performance.

6 . In the absence of reliable estimates of fracture length and conductivity, optimizing fracture treatment designs is many times not possible and design changes can be counter-productive. 


\begin{abstract}
Recent advances in hydraulic fracture mapping technologies have provided a wealth of information on the created fracture length in numerous geologic settings. Prior to such measurements, fracture length was estimated using "un-calibrated" fracture propagation models - but there was significant uncertainty in the results that cascaded into subsequent production analyses. However, we also need to understand how the created fracture length relates to the location of proppant in the fracture and the producing or effective length to evaluate well performance and improve stimulation designs. Unfortunately, the advanced fracture mapping technologies that today provide accurate measurements of the created fracture length cannot provide any insights (yet) into the propped and effective fracture lengths. Advanced production data analyses, pressure transient testing, and/or numerical reservoir modeling are required to determine the effective fracture length.

This paper begins with a comparison of the strengths, weaknesses, and limitations of fracture modeling, production data analysis (PDA), pressure transient analysis (PTA), and numerical reservoir modeling to estimate effective fracture length and conductivity. This work also evaluates how the complexities (in the hydraulic fracture) associated with non-Darcy flow, multi-phase flow, and complex fracture geometries affect the results from the various techniques. This work documents the significant differences in "effective" fracture length that, in many cases, can result from each technique and how these uncertainties can impact fracture treatment designs and field development decisions.

The paper concludes with several field case histories that illustrate the integration of multiple technologies to determine the created, propped, and effective fracture length. The case histories illustrate the dramatic differences in created and effective fracture length that can occur in some reservoirs, while also showing that in some cases effective fracture lengths can be very similar to the created length (and quite long). Integrating the results from multiple diagnostic techniques in a consistent and coherent manner can provide significant insights into created, propped, and effective fracture length that are otherwise unattainable from each technique alone.
\end{abstract}

\title{
Summary and Conclusions
}

The ability to reliably determine both created and effective hydraulic fracture length is essential to optimize stimulation designs and completion strategies. This paper highlights many of the limitations associated with non-unique analyses from fracture modeling, PDA, PTA, and numerical reservoir simulation. The examples presented illustrate these limitations, but also show how reliable solutions can be obtained by integrating the results from multiple diagnostic technologies. The following conclusions emphasize many of the issues associated with the application of fracture diagnostic technologies.

1. Fracture modeling (net pressure analysis) can result in non-unique interpretations of fracture geometry if the physics of fracture propagation are uncertain. However, fracture models can be calibrated using other diagnostic technologies (primarily microseismic and tiltmeter mapping), significantly reducing the uncertainty in predicted fracture geometry.

2. Production analyses and reservoir simulation history matching can result in non-unique interpretations of effective fracture length and conductivity in the absence of accurate measurements of reservoir permeability and pressure.

3. The combination of pre- and post-fracture pressure buildup tests can provide important information to evaluate hydraulic fracture performance. Pre-fracture PBU tests in low permeability reservoir can provide important measurements of permeability and pressure that significantly reduce the uncertainty in determining effective fracture length from subsequent production and post-fracture PBU analyses.

4. Microseismic and/or tilt fracture mapping can provide direct measurements of created fracture length and fracture complexity, but cannot provide insights into the propped and effective fracture length. This is a key limitation of current hydraulic fracture mapping technologies and emphasizes the need for comprehensive fracture modeling and production evaluation studies.

5. Combining multiple fracture diagnostic technologies and utilizing consistent evaluation procedures can provide a more reliable evaluation of hydraulic fracture performance, eliminating much of the uncertainty associated with non-unique solutions. 
6. In the absence of reliable estimates of created and effective fracture length and fracture conductivity, optimizing fracture treatment designs is many times not possible and design changes can be counter-productive.

7. Selecting the appropriate suite of diagnostic technologies and applying them with sufficient frequency can be critical to optimizing stimulation designs and field development strategies.

The above Abstract and Summary and Conclusions are taken from the Peer Approved revised version of IPTC 12147 [C.L. Cipolla, CARBO, E.P. Lolon, StrataGen Engineering, and M.J. Mayerhofer, Pinnacle]. The paper was originally prepared for presentation at the International Petroleum Technology Conference held in Kuala Lumpur, Malaysia, 3-5 December 2008. 